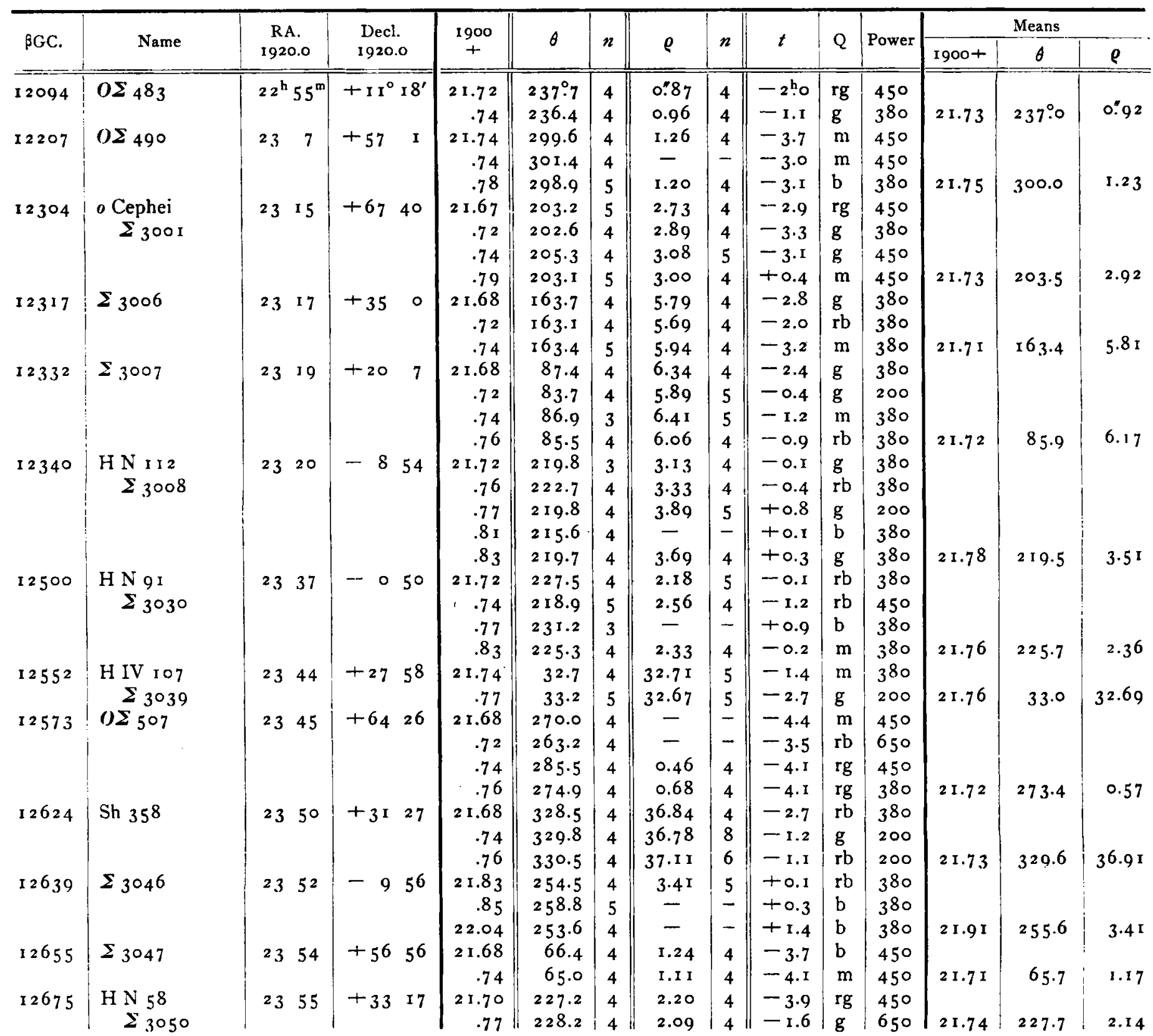

Kowloon, Elgin Rd., Sutton, Surrey, 1922 April 19.

W. Doberck.

Die Anwendung der Relativitätstheorie

auf die spektroskopischen Doppelsterne und die veränderlichen Sterne. Von G. Schain.

Nach der Relativitätstheorie müssen die Grundgleichungen der Mechanik durch einige Glieder ergänzt werden, die im Nenner das Quadrat der Lichtgeschwindigkeit enthalten; diese Korrektionen sind sehr klein und haben meistens nur theoretische Bedeutung. Je größer die Geschwindigkeit des Körpers und je größer die Masse des Anziehungskörpers ist, desto merklicher ist der Effekt des Gravitationsfeldes.

Die Rechtfertigung der Relativitätstheorie muß in solchen Erscheinungen gesucht werden, beidenen wir Geschwindigkeiten haben, die alle bisher im Sonnensystem bekannten übersteigen.
Deswegen habe ich es unternommen, diese Theorie auf die spektroskopischen Doppel- und veränderlichen Sterne kurzer Umlaufszeit anzuwenden.

Am meisten wirkt die Störung des Gravitationsfeldes auf die Perihellänge

$$
\mathrm{d} \omega=6 \pi k M /\left(c^{2} p\right)=24 \pi^{3} a^{2} /\left[T^{2} c^{2}\left(\mathrm{I}-c^{2}\right)\right] \text {. }
$$

Der Parameter enger Paare ist sehr klein, die Masse ist aber nach den Untersuchungen Ludendorffs im allgemeinen größer als die der Sonne; solche Systeme sind für diesen Zweck am geeignetsten. 
In der folgenden Tabelle gibt die zweite Spalte die Umlaufszeit; die dritte Spalte die Bewegung der Apsidenlinie für einen Umlauf; die vierte Spalte die Bewegung der Apsidenlinie für ein Jahr; die fünfte Spalte die Verschiebung der Epoche des Helligkeitsminimums für ro Jahre.

\begin{tabular}{|c|c|c|c|c|c|c|}
\hline U Ophiuchi & 0.80 & $2: 4$ & & I $7: 8$ & & ${ }^{n} 23^{s}$ \\
\hline RX Herculis & 0.90 & 2.1 & ० & 14.2 & 3 & 22 \\
\hline$\gamma$ Bootis & 0.29 & $4 \cdot 5$ & $\mathbf{I}$ & 33.2 & 7 & 9 \\
\hline $\mathrm{U} \cdot$ Pegasi & 0.37 & 4. I & $\mathbf{I}$ & 14.5 & 6 & 34 \\
\hline Algol & 2.87 & 3.7 & ० & $7 \cdot 5$ & 4 & 53 \\
\hline$\beta$ Canis maj. & 0.25 & 4.9 & 2 & 0.0 & & \\
\hline$\pi^{5}$ Orionis & 0.87 & 2.2 & 0 & I $5 \cdot 3$ & & \\
\hline V Puppis & $x .45$ & 15.4 & I & $4 \cdot 5$ & & \\
\hline RZ Cassiopeiae & 1.20 & 4.9 & ○ & 25.0 & 7 & 59 \\
\hline $\boldsymbol{\tau}$ Tauri & $1.5^{\circ}$ & 6.3 & ○ & $25 \cdot 5$ & & \\
\hline u Herculis & 2.05 & 18.5 & 0 & 54.9 & 7 & 28 \\
\hline
\end{tabular}

Bei den Systemen mit unbekannten Massen wurden diese der der Sonne gleich angenommen. Man müßte hier noch die Störungen, die von der Rotation herrühren, das sogenannte Thirringsche Korrektionsglied, anbringen, aber seiner Kleinheit halber kann es in erster Annäherung vernachlăssigt werden. Wir wollen jetzt den Sinn der letzten Spalte auseinandersetzen. Wir zerlegen die wahre Anomalie in eine Reihe nach den Potenzen der Exzentrizität

$$
v=M+2 e \sin M+5 / 4 e^{2} \sin 2 M+\cdots \text {. }
$$

Oder mit ziemlicher Annäherung

$$
M=v-2 e \sin v-5 / 4 e^{2} \sin 2 v \text {. }
$$

Statt der wahren und mittleren Anomalie führen wir die wahre und mittlere Länge ein, wobei wir nicht vom Knoten, sondern von der Projektion des Visionsradius auf die Bahnebene aus rechnen. Dann erhalten wir den Ausdruck

$$
\begin{gathered}
z_{0}=z-2 e \sin (z-\pi)-5 / 4 e^{2} \sin 2(z-\pi) \\
\text { wo } \quad z=90^{\circ}+w \quad z_{0}=n t+c \quad \pi=90^{\circ}+\alpha
\end{gathered}
$$

ist. Dadurch bekommen wir

$t=\left(-c+90^{\circ}\right) / n+w / n-2 e \sin (w-\alpha) \cdot / n-5 / 4 e^{2} \sin 2(w-\alpha) \cdot / n$ und für die Epoche des Minimums $w=2 m \pi$ oder $(2 m+1) \pi$ endgültig

$$
\begin{aligned}
& \quad t=T_{0}+m P+(e / \pi) P \sin \alpha+5 / 8\left(e^{2} / \pi\right) P \sin 2 \alpha \\
& \text { wo } n=2 \pi / P .
\end{aligned}
$$

Auf analoge Weise können wir ableiten, daß die Dauer der Finsternis sich ändert. Infolge der Apsidenbewegung verschiebt sich die Epoche des Minimums. Für einige Sterne beträgt die Apsidenbewegung in Io Jahren $20^{\circ}$ und mehr, eine Größe, die der Beobachtung durchaus zugänglich ist. Für diese Veränderlichen kann die Verschiebung der Epoche des Minimums ı o Minuten und mehr entsprechen. Das letztere hängt auch $\mathrm{ab}$ von der Lage des Perihels und von der Exzentrizität. ${ }^{1)}$ In unseren Rechnungen haben wir $\alpha=0$ gesetzt, d. h. den Maximalwert $\mathrm{d} t$ berechnet.

Der Beobachtung können keinesfalls to Minuten entgehen, insbesondere, wenn die Dauer der Finsternis gering ist und wir eine starke Senkung und Steigung der Helligkeitskurve haben (Y Leonis, RZ Cassiopeiae und andere).
Es ist nun die Frage, ob wir in Wirklichkeit bei den spektroskopischen Doppelsternen eine Apsidenbewegung und eine Abweichung der beobachteten Epoche des Minimums von der vorausberechneten finden.

Tatsächlich wurde ein ähnlicher Effekt an einigen engen Paaren beobachtet, und sogar mehr, die beobachtete Apsidenbewegung und Verschiebung der Epoche stellte sich oft größer heraus, als es die Relativitätstheorie erfordert ( $\beta$ Cephei, $\gamma$ Bootis, Algol, RZCassiopeiae und andere). Allerdings kennen wir die Masse der Himmelskörper nicht genau, um einen völlig bestimmten theoretischen Wert der Verschiebung zu erlangen. Aber auch wenn wir eine volle Übereinstimmung beobachten würden, so könnten wir doch nicht die Richtigkeit der Relativitätstheorie behaupten.

Der Grund liegt darin, daß, wenn wir beim Hauptstern eine Abplattung zulassen, wir damit eine Störungskraft einführen, welche die Apsidenlinie in direkte und die Knotenlinie in umgekehrte Bewegung bringt.

Der bekannte Ausdruck für den Zusammenhang der Abplattung des Zentralkörpers mit der Apsidenbewegung gibt $\mathrm{d} \pi=n(\varepsilon-1 / 2 \sigma)\left[M /\left(M_{1}+M\right)\right](R / a)^{2} \cdot 1 /\left(1-e^{3 / 2}\right) \cdot \cos i$.

Tisserand hat gezeigt, daß eine Abplattung $\varepsilon=1 / 288$ für das Algolsystem genügend ist für die Erklärung der Jahresbewegung der Apsidenlinie um $2: 5$. Ist der Körper unhomogen, so muß $\varepsilon$ größer sein.

Darin besteht eine der größten Schwierigkeiten der Anwendung der Relativitätstheorie, aber doch ist der Fall nicht hoffnungslos. Wir müssen eigentlich das Auftreten mehrerer Perioden in der Bewegung der Apsidenlinie beobachten, und mit Hilfe der harmonischen Analyse können dann alle Perioden getrennt werden. Alle gut bekannten veränderlichen Sterne zeigen einen komplizierten Charakter der Veränderung der Epoche des Minimums. Gewöhnlich bemerkt man außer dem periodischen Hauptglied auch andere mit besonderen Perioden.

Man kann endlich zulassen, daß nicht alle Sterne eine merkliche Abplattung besitzen. Dann kann der Effekt der Bewegung der Apsidenlinie ausschließlich der Relativitätstheorie zugeschrieben werden.

Die oben angefihrten Resultate beruhen auf der Annahme, daß die Masse der Trabanten recht gering ist; aber gerade in den engen Paaren kurzer Periode ist dieses nicht zulässig.

Die Differentialgleichungen der Bewegung nach der Relativitätstheorie ${ }^{2}$ ) können angenähert gelöst werden, wenn man die Störungskraft in drei Komponenten zerlegt, $W, S, T$. $\mathrm{Da}$ die Störungskraft nur in der Bahnebene wirkt, so ist $W=0$, folglich bleiben Knoten und Neigung unverändert.

Nach der Methode der Variation der Elemente erhalten wir für die anderen Elemente

$$
\begin{aligned}
\mathrm{d} a / \mathrm{d} t & =2 / n \cdot\left(\mathrm{I}-e^{2}\right)^{-1 / \omega} \cdot\left(S e \sin v+T^{1 / 2} p\right) \\
\mathrm{d} e / \mathrm{d} t & =\left(\mathrm{r}-e^{2}\right)^{1 / 2} / n a \cdot[S \sin v+T(\cos u+\cos v)] \\
e \mathrm{~d} \pi / \mathrm{d} t & =\left(\mathrm{r}-e^{2}\right)^{1 / 9} / n a \cdot[-S \cos v+T(\mathrm{r}+r \sin v / p)] .
\end{aligned}
$$

Führt man einige Proberechnungen aus und benutzt eine willkürliche Kombination der Komponentenmassen, so erkennt man, daß im allgemeinen die Masse des Trabanten

1) Für $\gamma$ Bootis, U Pegasi und RZ Cassiopeiae haben wir $e=0.20$ gesetzt.

2) Siehe Prof. de Sitter. MN 77.165. Die Differentialgleichungen Nr. (84). 
den Effekt der Apsidenlinie steigert. Aber gleichzeitig kommen wir zu einer anderen Störungskraft, nämlich zu einer Überlagerung zweier Gravitationsfelder. Sind die Größen der Körper gering im Vergleich mit ihrer gegenseitigen Entfernung, so ist der obengenannte Effekt nicht groß. Ist das nicht der Fall, was sehr oft bei den spektroskopischen Doppelsternen stattfindet, so hat die Überlagerung der Felder eine wesentliche Bedeutung. Dieses einer Berechnung zu unterziehen, ist analytisch recht schwer.

Pulkowo, 1922 April 5.
Gegenwärtig steht die Frage der Anwendbarkeit der Relativitätstheorie auf die spektroskopischen Doppel- und die veränderlichen Sterne noch offen. Es ist notwendig, eine möglichst große Zahl ähnlicher Systeme mit kurzer Periode zu erforschen. Berücksichtigt man die Masse des Systems und ergibt sich, daß die Reihe der periodischen Glieder nach Amplitude und Periode dem theoretischen Wert, der aus der Relativitätstheorie folgt, entspricht oder nicht entspricht, so erhält man damit eine positive oder negative Lösung des Problems.

\section{Systematische Unterschiede zwischen den Rektaszensionen des Katalogs Pulkowa 1915.0 und der Kataloge von Auwers, Boss, Pulkowa 1900.0 und Pulkowa 1905.0. Von F. Renz.}

Im Herbst I 9 I I begannen am Ertelschen Passageninstrument der Pulkowaer Sternwarte die Beobachtungen für einen Fundamentälkatalog von 1426 Sternen, die im Frühling I $916 \mathrm{im}$ großen und ganzen ais abgeschlossen betrachtet werden konnten, da nur noch vereinzelte Lücken auszufüllen waren. Dieser Katalog, der durch Verschmelzung der beiden Pulkowaer Kataloge von I 900 und I 905 entstanden war, bildet für den nördlichen Himmel den Hauptbestandteil der Sternliste, welche von der Fundamentalsternkommission des Comité permanent pour l'exécution de la Carte photographique du Ciel zusammengestellt ist. An den Beobachtungen am Pulkowaer Passageninstrument beteiligten sich außer mir zunächst die Herren Matkezitsch und Faschnoff, nach dem Abgang des letzteren aus Pulkowa im Sommer I 9 I 2 trat Herr Semenoff an seine Stelle. Jeder Stern wurde mindestens achtmal beobachtet und, wenn seine Deklination größer ist als $+45^{\circ}$, ebenso häufig in der unteren Kulmination. Die Zeitsterne, deren Zahl 3 I betrug, beobachteten wir an jedem klaren Tage vom fruhen Morgen bis nach Mitternacht, um eine etwaige Abhängigkeit der aus ihnen erhaltenen Uhrkorrektionen von der Tageszeit aufzudecken. Der Nullpunkt der Miren und die Korrektion der am Niveau abgelesenen Neigung wurden aus den Polsternen $\alpha$ und $\delta$ Ursae minoris in beiden Kulminationen und in den Lagen $O$ und $W$ des Instruments $a b$ geleitet. Die Positionen der Zeitsterne waren zunächst dem Berliner Jahrbuch entnommen. Später wurden ihre, aus den Beobachtungen folgenden, Verbesserungen nach der von Herrn Prof. Cohn vorgeschlagenen Methode von Herrn Faschnoff berechnet und die Örter der Katalogsterne dementsprechend korrigiert. Zur Ableitung der Korrektion des Äquinoktialpunktes wurde die Sonne, so oft das Wetter es gestattete, beobachtet, jedoch muß jch vorläufig auf eine Berechnung dieser Korrektion verzichten, weil die gleichzeitigen Beobachtungen der Sonne am Vertikalkreise mir nicht zugänglich sind. Abgesehen von diesem Fixpunkt stellt also der Katalog ein selbständiges System dar. Da seine Bearbeitung gegenwärtig abgeschlossen ist, der Druck des Manuskripts sich aber, aus von mir unabhängigen Gründen, verzögern dürfte, gebe ich zunächst nur eine Zusammenstellung der systematischen Abweichungen seiner Positionen von denen anderer Fundamentalkataloge. Seit Ausbruch des Krieges sind uns nur von wenigen Sternwarten Publikationen zugegangen; es ist $\mathrm{mir}$ daher nicht bekannt, welche Observatorien sich an der Beobachtung des Sternverzeichnisses des Comité permanent beteiligt haben und ob ihre Resultate veröffentlicht sind. Ich beschränke mich infolgedessen auf eine Vergleichung mit den Katalogen von Auwers-Peters, Boss, Pulkowa I 900.0 und Pulkowa I 905.0 .

Nachdem die Auwers-Petersschen Sternörter mit Benutzung des von Prof. Küstner angegebenen Koeffizienten 0.0052 auf die mittlere Helligkeit $4^{\mathrm{m}} \cdot \circ$ reduziert worden waren, wurden die Unterschiede zwischen Pulkowa I9 I 5.0 und den vier anderen Katalogen gebildet, nach Helligkeit, Rektaszension und Deklination gruppiert und in Mittelwerte zusammengezogen. Zur Ableitung $\operatorname{der} \boldsymbol{A} \boldsymbol{\alpha}_{\boldsymbol{\alpha}}$ und der Helligkeitsgleichung benutzte ich zunächst Sterne zwischen $-10^{\circ}$ und $+60^{\circ}$ Deklination. Die nördlicheren Sterne wurden später besonders behandelt. Eine zweite Näherung erwies sich nur in den Fällen als notwendig, wenn die Verteilung der Sterne innerhalb der betreffenden Gruppe sehr ungleichförmig war. Die dabei erhaltenen Verbesserungen der ersten Näherung überstiegen selten \pm 0.502 . Um den Gang der systematischen Unterschiede besser vergleichen $z u$ können, habe ich alle Kataloge durch Hinzufügung der an den Kopf einer jeden Spalte gestellten Zahlen auf das Äquinoktium des Auwers. Petersschen Katalogs bezogen, das auch dem Katalog Pulkowa I 9 I 5.0 vorläufig zugrunde gelegt ist.

Was die Helligkeitsgleichung anbetrifft, so läßt sich der neue Pulkowaer Katalog als frei davon betrachten, da die Beobachtungen mit dem selbstregistrierenden Mikrometer angestellt sind und die helleren Sterne durch drei Netzblenden, welche die Sterngrößen um 2, 4 und 6 Klassen abschwächten, auf eine mittlere Helligkeit von $5^{\mathrm{m}} \cdot 5$ bis $7^{\mathrm{m}} \cdot 5$ gebracht wurden.

Helligkeitskorrektion der Fundamentalkataloge von Auwers ${ }^{1}$ ), Boss, Pulkowa 1900 und 1905.

\begin{tabular}{|c|c|c|c|c|c|c|c|}
\hline Sterngröße & $\mathrm{P}_{15}-A(\mathrm{ko}$ & & $\begin{array}{l}P_{15}-L \\
\Delta \alpha\end{array}$ & $B$ & $\begin{array}{l}P_{15}-P_{C} \\
\Delta \alpha\end{array}$ & $*$ & $\begin{array}{l}P_{15}-P_{05} \\
\Delta \alpha\end{array}$ \\
\hline $\begin{array}{c}<2.0 \\
(\text { Mittel 1.4) }\end{array}$ & +0.001 & 28 & -0.002 & 28 & +0 .0 4 & 24 & +0.00127 \\
\hline $2.1-3.0$ & 0.000 & $6 \mathrm{I}$ & $-0.00 \mathrm{I}$ & $6_{3}$ & +0.014 & 46 & +0.004 \\
\hline $3.1-4.0$ & +0.001 & \pm 57 & +0.002 & 160 & +0.012 & 67 & 0.000160 \\
\hline $4.1-5.0$ & -0.002 & 87 & +0.002 & 135 & -0.005 & $4 \dot{5}$ & $0.000 \quad 9 \mathrm{I}$ \\
\hline $5.1-6.0$ & -0.002 & 69 & 0.000 & 283 & -0.004 & 207 & -0.003 \\
\hline $6.1-7.0$ & +0.005 & 10 & $-0.00 \mathrm{r}$ & 26 & -0.009 & 102 & o6] \\
\hline
\end{tabular}

Die Unterschiede $\mathrm{P}_{15}-A_{\text {korr. }}$ und $\mathrm{P}_{15}-B$ lassen keine $\mathrm{Ab}$ hängigkeit von der Sterngröße erkennen, obgleich sie wegen des von Boss angewandten, etwas größeren Helligkeitskoeffizienten vorhanden sein müßte. Der Pulkowaer Katalog i 905.0 